\title{
A Brain-Based Endophenotype for Major Depressive Disorder
}

\author{
Bradley S. Peterson and Myrna M. Weissman \\ Columbia College of Physicians \& Surgeons and New York State Psychiatric Institute, New York, \\ New York 10032 \\ Bradley S. Peterson: PetersoB@childpsych.columbia.edu; Myrna M. Weissman: weissman@childpsych.columbia.edu
}

\section{Abstract}

We have identified a brain-based endophenotype for major depressive disorder (MDD) that includes thinning of the cortex of the lateral aspect of the right hemisphere and the medial aspect of the left, as well as bilateral hypoplasia of frontal and parietal white matter. The endophenotype status of these abnormalities is supported by their presence in a multi-generational cohort of persons who themselves do not have MDD but who are at increased familial risk for developing the illness. Those who have the endophenotype but who are not ill nevertheless still suffer from inattention and poor visual memory for social stimuli in direct proportion to the magnitude of cortical thinning and white matter hypoplasia within the endophenotype. Identification of this endophenotype and its cognitive correlates provides targets for devising new preventive and therapeutic interventions for MDD.

\section{Keywords}

imaging; cortex; white matter; biomarker; attention; anxiety

\section{OPERATIONAL DEFINITION OF AN ENDOPHENOTYPE}

Immediately observable signs and symptoms of medical diseases rarely, if ever, relate directly to their underlying genetic, epigenetic, and environmental determinants. Much more often, differing pathogenic determinants instead map to similar, identical, or overlapping signs and symptoms. This kind of mapping is a general rule for psychiatric disorders, all of which are thought to have heterogeneous causes despite being defined by a single set of diagnostic criteria. Conversely, identical underlying causes, whether they are genes or environmental influences, can map to differing signs and symptoms. These complex mappings between causes and observable effects in behavioral phenotypes have hampered identification of the specific causes of psychiatric illnesses. Those difficulties have in turn motivated the search for "endophenotypes": entities that are measurable, though not visible to the unaided eye, and that lie along the causal chain from genes and environmental influences to their behavioral manifestations as symptoms. An endophenotype is an intermediate between a behavioral phenotype and its ultimate genetic and epigenetic determinants.

The criteria that operationally define an endophenotype vary to some extent across laboratories, with many using the term loosely to mean "disease correlate." But as originally

Copyright () 2011 by Annual Reviews. All rights reserved

DISCLOSURE STATEMENT

The authors are not aware of any affiliations, memberships, funding, or financial holdings that might be perceived as affecting the objectivity of this review. 
conceived and formulated to be most useful in identifying the genetic and epigenetic determinants of disease, the criteria are rigorous, requiring much more than simply the correlation of the putative marker with the presence of an illness. Indeed, in addition to being $(a)$ associated with an illness in the general population, a putative endophenotype must also be shown to be $(b)$ heritable, $(c)$ state independent (i.e., it must be present whether or not the illness is active), and $(d)$ found in unaffected family members of ill persons at a higher rate than in the general population. It must also $(e)$ cosegregate with the illness within families (1). These criteria clearly require that endophenotypes be sought within studies of multigenerational families containing both affected and unaffected individuals.

We have identified a brain-based endophenotype for major depressive disorder (MDD).

\section{PREVIOUS CANDIDATE ENDOPHENOTYPES}

Past imaging studies of MDD have assessed brain structure and function only in alreadyaffected individuals. Studies of already-affected individuals cannot discern whether differences from control populations represent the causes of MDD, compensatory responses that help to attenuate the severity of illness, the nonspecific effects of chronic illness or stress, or the effects of prior treatment (2). None have studied unaffected individuals who are at increased risk for developing MDD or multigenerational families of persons with MDD, and therefore none could validate the status of candidate endophenotypes. Despite these important caveats, those prior studies have emphasized the likely involvement of the limbic system and related frontal cortices in persons suffering from MDD (3), although the findings from those studies have generally had poor reproducibility and have reported relatively small effect sizes (4).

In addition to implicating the involvement of frontal cortex and limbic system, previous studies have suggested the possibility that the right hemisphere, particularly its posterior portion, is involved in the pathogenesis of MDD. Persons with MDD have been shown to exhibit selective impairment on right-hemisphere tasks $(5,6)$ and to have right-hemisphere deficits when performing tasks that employ the perception of lateralized stimuli (7-9). In addition, electroencephalographic (EEG) studies have reported reduced brain activity over the right posterior cortex in persons with either current (10) or remitted (11) MDD, as have studies of regional cerebral blood flow $(12,13)$. Taken together, these prior cross-sectional studies of already-affected individuals suggest that reasonable candidate brain regions for an MDD phenotype would include frontal cortex, the cortex of the right posterior hemisphere, and components of the limbic system.

\section{THE NEUROANATOMICAL FEATURES OF AN MDD ENDOPHENOTYPE}

We used magnetic resonance imaging (MRI) in a multigenerational cohort of individuals who were either affected or unaffected with MDD to search for a brain-based endophenotype (14). The first generation in this multigenerational cohort was identified as being either a clinically identified sample of adults who had MDD that was recurrent, moderate to severe, and functionally debilitating, or else a matched comparison group of individuals and their spouses from the same community who had no discernible lifetime history of MDD or other psychiatric illness $(15,16)$. Each of these groups in Generation 1 (G1) had children who were followed until adulthood as Generation 2 (G2), and those individuals in turn had children who were followed until adulthood as Generation 3 (G3). These three generations were followed longitudinally over five waves of assessment for the new onset and presence of psychiatric diagnoses. Those longitudinal assessments in this cohort $(15)$ and similar two-generation studies $(17,18)$ have shown that chronic severe depression is familial, with offspring of depressed individuals having a three- to five-fold increased risk of developing MDD themselves, and they helped to define the natural history 
of familial MDD, which tends to present initially with elevated rates of anxiety disorders before puberty, transforming to MDD beginning in mid to late adolescence. These multigenerational family studies have also shown that familial MDD tends to have an earlier onset and to be more severe, more recurrent, and less responsive to treatment than is nonfamilial MDD.

We obtained anatomical MRIs in 131 members of the second and third generations of this multigenerational cohort. We defined as being at "high familial risk" for developing MDD those members of G2 and G3 who were biological descendants of the MDD group in G1. Those defined as being at "low familial risk" were the G2 and G3 biological descendants of the unaffected control group in G1. The high- and low-risk groups contained 66 and 65 individuals, respectively. As expected, the frequencies of lifetime MDD and anxiety disorders were significantly greater in the high-risk group than in the low-risk group (56\% versus $23 \%$ for MDD; $52 \%$ versus $21 \%$ for anxiety disorders). Severity ratings of depression and anxiety symptoms at the time of the MRI scan were similar across groups, however, reflecting the fact that most of the participants in both of the risk groups were not in an MDD episode at the time of scan.

The anatomical images and the methods used to process them permitted detailed comparisons of two measures of brain structure across the high- and low-risk groups (19). One was a measure of the thickness of the cortical mantle, the $\sim 6-\mathrm{mm}$-thick layer of gray matter at the surface of the brain that contains most of the nerve cell bodies and neuropil (axonal and dendritic arbors and synapses) and that is the locus of most of the computational functions in the brain. Cortical thickness was compared across groups at each millimetersized point on the cerebral surface. The other was a measure of local volumes that permitted comparison of those volumes at each millimeter-sized cube throughout the threedimensional substance of the brain. We plotted in color the results of the statistical comparisons at each of these points.

These analyses revealed thinning of the cortical mantle over the lateral convexity of the right hemisphere and the medial surface of the left hemisphere in the high-risk group. The thinning in both of these regions was massive in its spatial extent, extending from the back of the brain to the front, sparing only an anterior portion of the surface of the right temporal cortex (Figure 1a). It was also massive in its magnitude within each of those broad cortical regions, with reductions in thickness averaging nearly $30 \%$. In contrast, areas of local thickening were also detected in the high-risk group in the cingulate cortex along the medial wall of the right cerebral hemisphere, especially the ventral anterior and posterior cingulate cortices. Thickening was also detected in the medial orbitofrontal cortex in the same hemisphere.

The analyses also revealed prominent volume reductions in the white matter of the frontal and parietal lobes bilaterally (Figure 2) (M.J. Dubin, M.M. Weissman, D. Xu, et al., manuscript submitted). We determined that the white matter fiber tracts most likely to pass through the regions of frontal and parietal hypoplasia were the superior longitudinal fasciculus (21), the anterior half of the corpus callosum, and the cortical projections with the thalamus, brainstem, and spinal tracts. Of these various tracts, the superior longitudinal fasciculus is the one that passes through the regions of frontal and parietal hypoplasia and that connects the dorsal frontal cortex to the posterior temporal and parietal cortices, regions where cortical thinning was most prominent in the high-risk group. This fiber tract therefore could produce circuit-based disturbances that would associate the frontal and parietal white matter hypoplasia with the pattern of cortical thinning that we observed. We were interested in knowing whether the findings of cortical thinning and white matter hypoplasia were 
present in the same people and to the same extent, or whether these represented two distinct anatomical abnormalities within the high-risk population.

We therefore assessed whether these two regional disturbances in the cortex and white matter were correlated, and therefore were both portions of the same underlying putative endophenotype. We performed two complementary correlation analyses, one that correlated a single average measure of cortical thickness for each gyrus of the right hemisphere with local volumes at each point of the white matter, and another that correlated a single average measure of local volume in frontal white matter with measures of thickness at each point of the right-hemisphere cortex. Both analyses provided overwhelming evidence that the cortical thinning and white matter hypoplasia are highly correlated and part of the same underlying endophenotype (Figure 3). Moreover, these analyses showed that cortical thickness and white matter volumes are highly intercorrelated even within the low-risk group alone, suggesting that these measures probably covary strongly even in the general population, and that they are likely components of a single circuit or set of brain circuits. Nearly identical correlations in the high-risk group alone then suggested that this set of circuits was hypoplastic within the high-risk group. In other words, our findings of reduced cortical thickness and reduced white matter volumes likely derived from the hypoplasia of an entire circuit or set of circuits in the high-risk group. Despite the strong intercorrelations of cortical thinning with white matter hypoplasia, we emphasize this set of findings could not formally assess and identify a direction of causality to determine whether the processes responsible for cortical thinning caused the white matter tract abnormalities, whether the white matter abnormalities caused the cortical thinning, or whether some pathological process produced a hypoplasia throughout the entire circuit, including both the cortex and white matter tracts that interconnect them.

\section{EVIDENCE THAT THIS CIRCUIT-BASED HYPOPLASIA IS AN MDD ENDOPHENOTYPE}

To support the status of cortical thinning and white matter hypoplasia as components of a putative endophenotype for familial MDD, we assessed whether these features were present in members of the high-risk group who themselves had never been ill with either an anxiety disorder or MDD. We therefore compared never-ill persons in the high-risk group with never-ill persons in the low-risk group and detected cortical thinning and white matter hypoplasia in both groups that were virtually identical to our findings in the entire cohort (Figure $1 b$ ). These findings satisfied one of the strongest operational criteria for an endophenotype: its presence in unaffected family members. Moreover, we detected the same disturbances in both the youngest children and oldest adults of the cohort, indicating that the endophenotype is present throughout the lifespan, even in young children.

Taken together, these findings demonstrate that hypoplasia and thinning are not the consequences of having anxiety or MDD or of having received treatment for those illnesses. They are present in both affected and unaffected individuals at high familial risk, and they are established early in life. Because the findings are present even in young children, they are unlikely to be degenerative in nature and likely represent a disturbance of normal brain development that predisposes to MDD. Whether the circuit-based disturbances are the consequences of genetic, epigenetic, or environmental effects or their combination we cannot yet say, although both genetic and environmental factors and their interactions are known to influence the risk for developing MDD (22). Being born into a family with depressed parents is known to have a profound effect on the behavior of children $(23,24)$, and the altered interactions with depressed caregivers could potentially produce enduring changes in neural systems, particularly those that subserve the processing of social and emotional information coming from caregivers and the larger environment. 


\section{NORMAL FUNCTIONS OF THE CIRCUITS THAT COMPOSE THIS ENDOPHENOTYPE}

Decades of clinical and preclinical research in healthy and ill individuals have suggested that the brain regions and neural circuits that compose this endophenotype support attentional and social processing. The right hemisphere, for example, has long been thought to subserve certain forms of attention, arousal, and vigilance (25), particularly the perception of social and emotional cues from the environment in both the visual and verbal domains. Substantial support for this claim comes from psychological and lesion studies in humans and nonhuman primates, which have suggested that the right hemisphere is dominant for the processing and recall of emotional faces, emotion-denoting words, linguistic prosody, and emotional meaning (26-32).

The cortical component of the MDD endophenotype also includes thinning of the medial wall of the left hemisphere and thickening of the cingulate and orbitofrontal cortices of the right hemisphere. The medial wall of the cerebral surface includes the medial prefrontal cortex, which helps to regulate emotions (33), and the ventral anterior and posterior cingulate cortices, which likely contribute to the emotional quality of free associative thought (34), internally focused thought (35), and episodic memories (36). The cingulate cortex has numerous components (37), and its subgenual portion in particular is thought to participate in emotional processing (3). The orbitofrontal cortex participates in the processing of reward and the hedonic qualities of learned experience (38). The cingulate and orbitofrontal cortices are important components of the limbic system and are intimately connected to other limbic structures, such as the amygdala, hypothalamus, and brainstem autonomic nuclei (3).

The white matter component of the MDD endophenotype connects frontal and parietal cortices via the superior longitudinal fasciculus to support attentional, arousal, and vigilance processes (39). It also contains pathways that connect the anterior cingulate, parahippocampal, and entorhinal cortices with the amygdala, hippocampus, hypothalamus, and anterior nucleus of the thalamus to support the processing and regulation of emotions.

\section{THE COGNITIVE CONSEQUENCES OF HAVING THIS ENDOPHENOTYPE}

The fact that many individuals in the high-risk group who have the circuit-based endophenotype may by definition never have been diagnosed with an anxiety disorder or MDD does not mean that they are free of all cognitive and behavioral problems. Indeed, we found that to the degree that they had this circuit-based abnormality, they also had substantial problems with attention (e.g., staying focused on tasks, organizing their activities, and filtering out distracting stimuli) and memory for visual social stimuli (e.g., facial identification and social scenes on standardized memory scales). Perhaps most remarkably, the correlation of cortical thickness and white matter volumes with measures of attention and memory for social stimuli were highly significant not only in the high-risk group, but also in the low-risk group separately. The locations of significant correlations were identical to the locations of the endophenotype-with greater cognitive impairment accompanying thinner cortices along the lateral surface of the right hemisphere and the medial wall of the left, with thicker cortices in the subgenual and orbitofrontal cortices, and with smaller volumes in frontal white matter (Figure 1c,d; Figure $2 b$ ).

The spatial pattern of these correlations and their presence even in the low-risk group suggest that neural circuits connect the frontal and parietal white matter with the lateral surface of the right hemisphere and medial wall of the left, consistent with the known connectivity of white matter fiber tracts within the white matter portions of this 
endophenotype. The thinner the cortices and the smaller the volumes of white matter are within this circuit, the poorer are an individual's capacities for sustained attention and processing of social stimuli. This seems to be a truth in the general population, given that the relationship was present in both risk groups of our sample. The weight of the evidence suggests that attentional and social processing capacities in the high-risk group were on average poorer than in the low-risk group because the high-risk group on average had reduced cortical thicknesses and white matter volumes within this circuit.

We speculate that the cognitive difficulties that accompany hypoplasia within this circuitbased endophenotype are what place individuals at risk for developing an anxiety disorder or MDD. Attentional capacities are components of executive functions that are vital for regulating thoughts, emotions, and behaviors, and difficulties in these areas will inherently limit an individual's overall functional capacity and real-world efficacy. Disturbances in processing of emotionally and socially relevant stimuli within a dysfunctional right hemisphere could predispose an individual to emotional disturbances within an interpersonal world (40) by reducing the interpersonal support and social relationships that would otherwise redress problems associated with impairments in attentional and self-regulatory capacities.

Further evidence that hypoplasia in these circuits caused the cognitive disturbances associated with this MDD endophenotype came from novel mediator analyses applied to the imaging measures at each point of the brain. These analyses demonstrated that cortical thinning mediated the association of familial risk with attentional problems and difficulty remembering social stimuli, and that these cognitive difficulties in turn mediated the association of cortical thinning with risk for depression. In other words, familial predisposition leads to cortical thinning, which leads to cognitive problems, which in turn lead to the symptoms of MDD.

\section{ELECTROPHYSIOLOGICAL CORRELATES OF THIS ENDOPHENOTYPE}

A prior EEG study of our cohort demonstrated greater alpha asymmetry of the EEG in posterior leads of children and adults in G2 who had two depressed parents than in persons with one or no depressed parents (41). The increased asymmetry derived from relatively less activity in parietotemporal leads over the right hemisphere and was independent of a personal lifetime history of MDD, consistent with prior EEG studies reporting right-sided hypoactivation of parietotemporal cortices in numerous patient populations, including adolescents and adults with MDD (42-45), previously depressed but currently euthymic adults (11), and children with low positive emotionality (46). This reduced electrophysiological activity in the right posterior cortices has been most consistently interpreted as representing disturbances in the autonomic, cognitive, and behavioral aspects of emotional arousal (47-49).

The presence and location of reduced right parietotemporal activity in those who have higher familial risk but no personal history of MDD, and in those with remitted illness, suggest that reduced right parietotemporal activity could be a trait marker for vulnerability to MDD and therefore an electrophysiological correlate of our MDD endophenotype. We assessed how tightly correlated alpha activity was with the thickness of the underlying cortex in the 75 persons from our cohort who had both MRI and EEG measures. Alpha power in the EEG correlated inversely with cortical thickness in both the high- and low-risk groups, particularly over the right posterior cortex, indicating that progressively greater alpha power (less activity) accompanied progressively thinner cortices (G.E. Bruder, R. Bansal, C.E. Tenke, et al., manuscript submitted). This finding suggests that cortical thinning likely accounted for the reduced EEG activity in our high-risk group. 
Consistent with the evidence that the right hemisphere subserves attention, arousal, and vigilance, a prior study of startle amplitude in a subset of persons in G2 and G3 of this cohort reported an exaggerated startle response in the high-compared with the low-risk group (51), suggesting that exaggerated phasic arousal in these individuals in response to abrupt, startling stimuli may be linked to these right-hemisphere abnormalities (52) and may have contributed to the disturbances in attention that these participants reported. Our interpretation that cortical thinning disrupts arousal processes based within the right hemisphere is also consistent with a magnetoencephalography study that reported reduced activity over the right posterior cortex in persons with MDD during the viewing of emotionally arousing pictures (53).

\section{TRIGGERING STATE-RELATED ILLNESS FROM THIS ENDOPHENOTYPE}

Persons who carry this circuit-based endophenotype are at increased risk for developing MDD, presumably by virtue of the effects that the endophenotype has on attentional, social, and emotional processing. Something in addition to this trait-related disturbance, however, is needed to convert this vulnerability into an overt disorder. Evidence from our multigenerational longitudinal cohort suggests that this trigger is cortical thinning in analogous portions of the hemisphere opposite the location of the MDD trait vulnerability. We detected highly significant correlations of cortical thickness with the severity of anxiety and depressive symptoms at the time of the MRI scan that were located in the posterior portion of the left hemisphere and the medial wall of the right (Figure 4). One of the hypothesized evolutionary advantages of having a bicameral brain is the functional reserve that a second hemisphere provides when illness or dysfunction affects one of the hemispheres. Therefore, we suspect that involvement of a circuit in the mirror-image location of our MDD endophenotype reduces functional reserve for attentional, social, and emotional processing that would otherwise attenuate or compensate for disturbances that MDD confers in those functional domains. In other words, the vulnerability to developing MDD is based primarily in right-hemisphere systems, but the primary determinant for actually being ill is the added, analogous disturbance in the left hemisphere.

\section{THE POTENTIAL CLINICAL AND RESEARCH UTILITY OF AN MDD ENDOPHENOTYPE}

The successful identification of an MDD endophenotype according to rigorous operational criteria has potentially important and far-reaching clinical and research implications. First, if the endophenotype is shown to be specific to MDD and not to be present in other disorders, it could help improve diagnostic classification that can be difficult when the signs and symptoms of MDD overlap those of other diagnoses or when the differential diagnosis includes both unipolar and bipolar MDD. Ultimately, the identification of an MDD endophenotype may supplement or, less likely, even replace psychiatric diagnosis on the basis of signs and symptoms. Second, because endophenotypes are by definition present prior to the onset of illness, this MDD endophenotype can be used as an aid in identifying persons who are at elevated risk for becoming ill and for the delivery of preventive interventions. Third, identification of the MDD endophenotype may help to identify a biological subype of this illness, which could then possibly aid in predicting the clinical course of the illness, individualizing treatment, and predicting therapeutic response. Determining whether treatment can modify this brain-based endophenotype for MDD and subsequent risk for becoming ill will be important. Fourth, this MDD endophenotype may aid in the search for more precise genetic and environmental determinants of MDD, given that endophenotypes are thought to be closer than psychiatric symptoms to the genetic and environmental determinants of illness. Finally, this MDD endophenotype should aid in the construction and validation of animal models for this illness, as animal models should 
recapitulate damage in the same neural systems that compose the human MDD endophenotype. We have studies under way in each one of these areas in an attempt to exploit the value of having identified an MDD endophenotype.

\section{Acknowledgments}

This work was supported in part by National Institute of Mental Health grants MH36197, 1P50MH090966, and K02-74677, grants from the National Alliance for Research in Schizophrenia and Affective Disorders, a National Institute for Drug Abuse supplement to grant MH36197, and the Sackler Institute at Columbia University. We thank our many collaborators, research staff, and families who have participated in this work. Without them, the progress reported in this paper would not have been possible.

\section{Glossary}

Endophenotype

MDD a measurable entity, not observable to the unaided eye, that lies along the causal chain from genes and environmental influences to their behavioral manifestations as symptoms. An intermediate between a behavioral phenotype and its ultimate genetic and epigenetic determinants

major depressive disorder

\section{LITERATURE CITED}

1. Gottesman II, Gould TD. The endophenotype concept in psychiatry: etymology and strategic intentions. Am J Psychiatry. 2003; 160:636-45. [PubMed: 12668349]

2. Peterson BS. Conceptual, methodological, and statistical challenges in brain imaging studies of developmentally based psychopathologies. Dev Psychopathol. 2003; 15:811-32. [PubMed: 14582941]

3. Price JL, Drevets WC. Neurocircuitry of mood disorders. Neuropsychopharmacology. 2010; 35:192-216. [PubMed: 19693001]

4. Murphy FC, Nimmo-Smith I, Lawrence AD. Functional neuroanatomy of emotions: a metaanalysis. Cogn Affect Behav Neurosci. 2003; 3:207-33. [PubMed: 14672157]

5. Rubinow DR, Post RM. Impaired recognition of affect in facial expression in depressed patients. Biol Psychiatry. 1992; 31:947-53. [PubMed: 1637932]

6. Silberman EK, Weingartner H, Post RM. Thinking disorder in depression. Arch Gen Psychiatry. 1983; 40:775-80. [PubMed: 6860078]

7. Levy J, Heller W, Banich MT, et al. Asymmetry of perception in free viewing of chimeric faces. Brain Cogn. 1983; 2:404-19. [PubMed: 6546034]

8. Jaeger J, Borod JC, Peselow E. Depressed patients have atypical hemispace biases in the perception of emotional chimeric faces. J Abnorm Psychol. 1987; 96:321-24. [PubMed: 3693681]

9. Bruder GE, Quitkin FM, Stewart JW, et al. Cerebral laterality and depression: differences in perceptual asymmetry among diagnostic subtypes. J Abnorm Psychol. 1989; 98:177-86. [PubMed: 2708661]

10. Deldin PJ, Keller J, Gergen JA, et al. Right-posterior face processing anomaly in depression. J Abnorm Psychol. 2000; 109:116-21. [PubMed: 10740942]

11. Henriques JB, Davidson RJ. Regional brain electrical asymmetries discriminate between previously depressed and healthy control subjects. J Abnorm Psychol. 1990; 99:22-31. [PubMed: 2307762]

12. Post RM, DeLisi LE, Holcomb HH, et al. Glucose utilization in the temporal cortex of affectively ill patients: positron emission tomography. Biol Psychiatry. 1987; 22:545-53. [PubMed: 3495297]

13. Uytdenhoef P, Portelange P, Jacquy J, et al. Regional cerebral blood flow and lateralized hemispheric dysfunction in depression. Br J Psychiatry. 1983; 143:128-32. [PubMed: 6616114] 
14. Peterson BS, Warner V, Bansal R, et al. Cortical thinning in persons at increased familial risk for major depression. Proc Natl Acad Sci USA. 2009; 106:6273-78. [PubMed: 19329490]

15. Weissman MM, Wickramaratne P, Nomura Y, et al. Offspring of depressed parents: 20 years later. Am J Psychiatry. 2006; 163:1001-8. [PubMed: 16741200]

16. Weissman MM, Wickramaratne P, Nomura Y, et al. Families at high and low risk for depression: a 3-generation study. Arch Gen Psychiatry. 2005; 62:29-36. [PubMed: 15630070]

17. Lieb R, Isensee B, Hofler M, et al. Parental major depression and the risk of depression and other mental disorders in offspring: a prospective-longitudinal community study. Arch Gen Psychiatry. 2002; 59:365-74. [PubMed: 11926937]

18. Hammen C, Burge D, Burney E, et al. Longitudinal study of diagnoses in children of women with unipolar and bipolar affective disorder. Arch Gen Psychiatry. 1990; 47:1112-17. [PubMed: 2244796]

19. Peterson BS. Form determines function: new methods for identifying the neuroanatomical loci of circuit-based disturbances in childhood disorders. J Am Acad Child Adolesc Psychiatry. 2010; 49:533-38. [PubMed: 20494263]

20. Deleted in proof

21. Petrides M, Pandya DN. Projections to the frontal cortex from the posterior parietal region in the rhesus monkey. J Comp Neurol. 1984; 228:105-16. [PubMed: 6480903]

22. Caspi A, Sugden K, Moffitt TE, et al. Influence of life stress on depression: moderation by a polymorphism in the 5-HTT gene. Science. 2003; 301:386-89. [PubMed: 12869766]

23. Tronick E, Reck C. Infants of depressed mothers. Harvard Rev Psychiatry. 2009; 17:147-56.

24. Ramchandani P, Psychogiou L. Paternal psychiatric disorders and children's psychosocial development. Lancet. 2009; 374:646-53. [PubMed: 19411102]

25. Posner MI, Petersen SE. The attention system of the human brain. Annu Rev Neurosci. 1990; 13:25-42. [PubMed: 2183676]

26. Sato W, Aoki S. Right hemispheric dominance in processing of unconscious negative emotion. Brain Cogn. 2006; 62:261-66. [PubMed: 16899333]

27. Ross ED, Harney JH, deLacoste-Utamsing C, et al. How the brain integrates affective and propositional language into a unified behavioral function. Hypothesis based on clinicoanatomic evidence. Arch Neurol. 1981; 38:745-48. [PubMed: 7316839]

28. Heilman KM, Gilmore RL. Cortical influences in emotion. J Clin Neurophysiol. 1998; 15:409-23. [PubMed: 9821068]

29. Bowers D, Blonder LX, Feinberg T, et al. Differential impact of right and left hemisphere lesions on facial emotion and object imagery. Brain. 1991; 114(Pt 6):2593-609. [PubMed: 1782533]

30. Adolphs R, Damasio H, Tranel D, et al. Cortical systems for the recognition of emotion in facial expressions. J Neurosci. 1996; 16:7678-87. [PubMed: 8922424]

31. Borod, JC.; Zgaljardic, D.; Tabert, MH., et al. Asymmetries of emotional perception and expression in normal adults. In: Gainotti, G., editor. Handbook of Neuropsychology. Amsterdam: Elsevier; 2001. p. 181-205.

32. Fernandez-Carriba S, Loeches A, Morcillo A, et al. Functional asymmetry of emotions in primates: new findings in chimpanzees. Brain Res Bull. 2002; 57:561-64. [PubMed: 11923029]

33. Drevets WC, Price JL, Furey ML. Brain structural and functional abnormalities in mood disorders: implications for neurocircuitry models of depression. Brain Struct Funct. 2008; 213:93-118. [PubMed: 18704495]

34. Mason MF, Norton MI, Van Horn JD, et al. Wandering minds: the default network and stimulusindependent thought. Science. 2007; 315:393-95. [PubMed: 17234951]

35. Lieberman MD. Social cognitive neuroscience: a review of core processes. Annu Rev Psychol. 2007; 58:259-89. [PubMed: 17002553]

36. Marsh R, Hao X, Xu D, et al. A virtual reality-based fMRI study of reward-based spatial learning. Neuropsychologia. 2010; 48:2912-21. [PubMed: 20570684]

37. Vogt BA, Finch DM, Olson CR. Functional heterogeneity in cingulate cortex: the anterior executive and posterior evaluative regions. Cereb Cortex. 1992; 2:435-43. [PubMed: 1477524] 
38. Hare TA, O'Doherty J, Camerer CF, et al. Dissociating the role of the orbitofrontal cortex and the striatum in the computation of goal values and prediction errors. J Neurosci. 2008; 28:5623-30. [PubMed: 18509023]

39. Posner MI, Rothbart MK. Toward a physical basis of attention and self regulation. Phys Life Rev. 2009; 6:103-20. [PubMed: 20161073]

40. Van Lancker D. Personal relevance and the human right hemisphere. Brain Cogn. 1991; 17:64-92. [PubMed: 1781982]

41. Bruder GE, Tenke CE, Warner V, et al. Electroencephalographic measures of regional hemispheric activity in offspring at risk for depressive disorders. Biol Psychiatry. 2005; 57:328-35. [PubMed: 15705347]

42. Bruder GE, Fong R, Tenke CE, et al. Regional brain asymmetries in major depression with or without an anxiety disorder: a quantitative electroencephalographic study. Biol Psychiatry. 1997; 41:939-48. [PubMed: 9110099]

43. Davidson RJ, Chapman JP, Chapman LJ. Task-dependent EEG asymmetry discriminates between depressed and nondepressed subjects. Psychophysiology. 1987; 24:585.

44. Kentgen LM, Tenke CE, Pine DS, et al. Electroencephalographic asymmetries in adolescents with major depression: influence of comorbidity with anxiety disorders. J Abnorm Psychol. 2000; 109:797-802. [PubMed: 11196007]

45. Reid SA, Duke LM, Allen JJ. Resting frontal electroencephalographic asymmetry in depression: inconsistencies suggest the need to identify mediating factors. Psychophysiology. 1998; 35:389_ 404. [PubMed: 9643053]

46. Shankman SA, Tenke CE, Bruder GE, et al. Low positive emotionality in young children: association with EEG asymmetry. Dev Psychopathol. 2005; 17:85-98. [PubMed: 15971761]

47. Heller W, Etienne MA, Miller GA. Patterns of perceptual asymmetry in depression and anxiety: implications for neuropsychological models of emotion and psychopathology. J Abnorm Psychol. 1995; 104:327-33. [PubMed: 7790634]

48. Posner J, Russell JA, Peterson BS. The circumplex model of affect: an integrative approach to affective neuroscience, cognitive development, and psychopathology. Dev Psychopathol. 2005; 17:715-34. [PubMed: 16262989]

49. Russell JA. Core affect and the psychological construction of emotion. Psychol Rev. 2003; 110:145-72. [PubMed: 12529060]

50. Deleted in proof

51. Grillon C, Warner V, Hille J, et al. Families at high and low risk for depression: a three-generation startle study. Biol Psychiatry. 2005; 57:953-60. [PubMed: 15860334]

52. Heller W, Nitschke JB, Etienne MA, et al. Patterns of regional brain activity differentiate types of anxiety. J Abnorm Psychol. 1997; 106:376-85. [PubMed: 9241939]

53. Moratti S, Rubio G, Campo P, et al. Hypofunction of right temporoparietal cortex during emotional arousal in depression. Arch Gen Psychiatry. 2008; 65:532-41. [PubMed: 18458205] 


\section{SUMMARY POINTS}

1. We have identified a brain-based endophenotype for major depressive disorder (MDD). It consists of cortical thinning of the lateral aspect of the right hemisphere and the medial aspect of the left, along with bilateral hypoplasia of the frontal and parietal white matter.

2. The endophenotype is present not only in persons who have MDD, but also in their unaffected family members at higher rates than in persons who are at low familial risk for MDD.

3. Those who have the endophenotype, but who are not ill, nevertheless suffer from inattention and poor visual memory for social stimuli in direct proportion to the magnitude of cortical thinning and white matter hypoplasia.

4. Measures of cortical thickness correlate significantly with local white matter volumes within the spatial domain of the endophenotype. This is true in persons at either high or low familial risk for MDD, suggesting that these white matter and cortical regions compose a neural circuit that is hypoplastic throughout both its components in those who have the MDD endophenotype.

5. Cortical thinning in the lateral surface of the left hemisphere and medial surface of the right - in the mirror-image locations of the MDD endophenotype-in individuals who have the MDD endophenotype seems to convert their vulnerability to developing MDD into an overt disorder.

6. Identification of this endophenotype and its cognitive correlates provides targets for devising new preventive and therapeutic interventions for MDD. 


\section{FUTURE ISSUES}

1. Identifying functional disturbances in brain tissue associated with this endophenotype.

2. Improving characterization of the white matter portions of the circuits that compose this endophenotype by using diffusion tensor imaging and magnetic resonance spectroscopy.

3. Determining the underlying cellular and molecular correlates of this endophenotype in post mortem studies of persons with familial MDD by using combined histological and imaging techniques.

4. Improving assessment of the cognitive, affective, and behavioral correlates of this endophenotype even in the absence of illness by using the techniques of cognitive and affective neuroscience.

5. Determining whether the endophenotype can be modified by pharmacological or psychological treatments.

6. Using the endophenotype to improve diagnosis and to individualize treatment.

7. Using the endophenotype to aid in the prevention of MDD. 

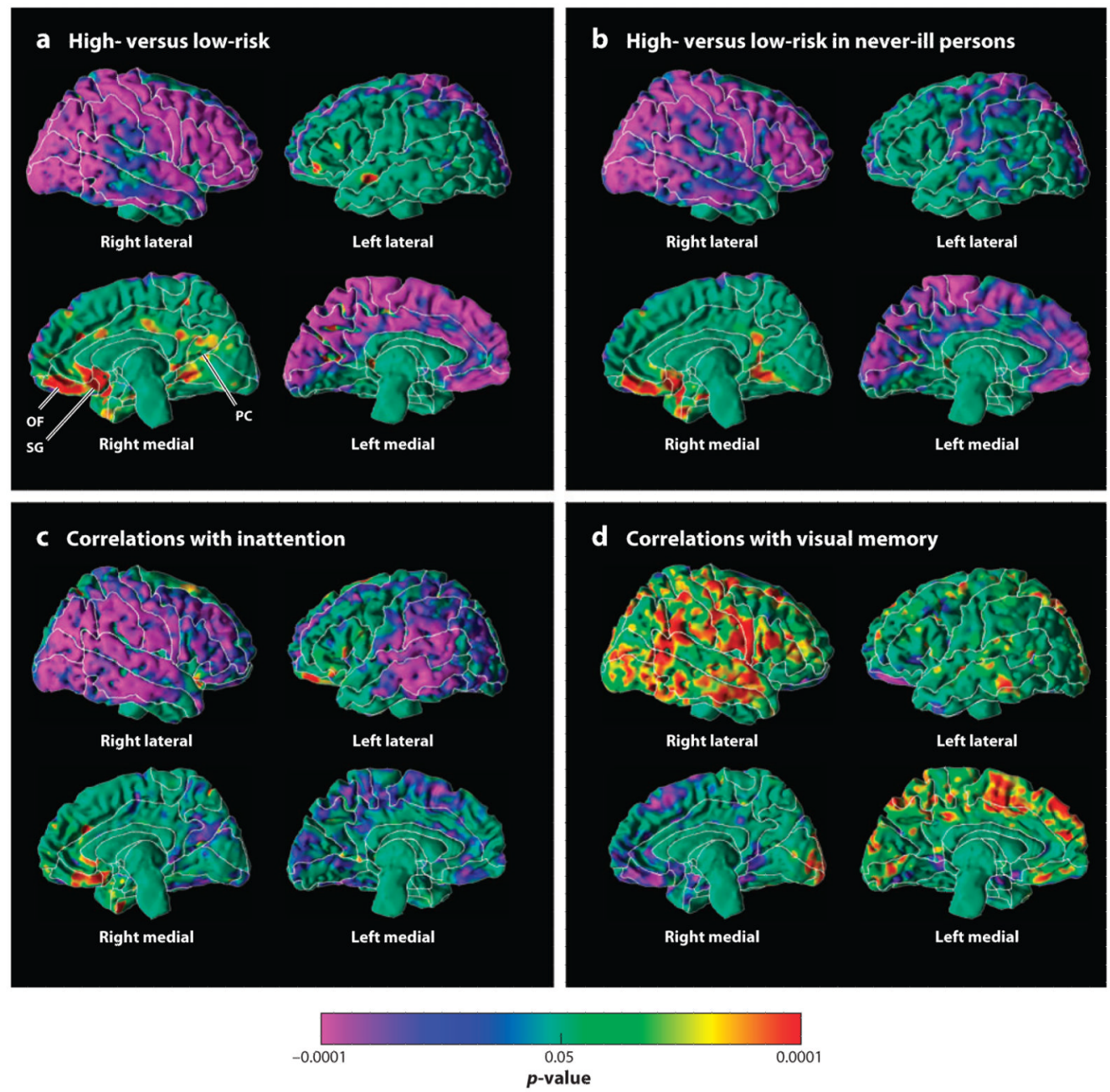

Figure 1.

Maps of cortical thickness. The statistical significance (probability values) of analyses of cortical thickness are color-coded at each point of the cerebral surface. The color bar indicates the color-coding of $p$-values for tests of statistical significance at each point of the brain. All statistical models covaried for age and sex. (a) Cortical thickness is compared at each point across all 131 members of the high- and low-risk groups. Warm colors (yellow, orange, and red) represent significantly thicker cortices and cooler colors (blue and purple) represent thinner cortices in the high-risk group. Significant thinning is present along the lateral aspect of the right hemisphere and the medial wall of the left. Significant thickening is present in the ventral anterior and posterior cingulate cortices, as well as the orbitofrontal cortex, along the medial wall of the right hemisphere. (b) Cortical thickness is compared across high- and low-risk groups but only in those participants $(n=79)$ who had no lifetime history of major depressive disorder (MDD). The pattern of cortical thinning is clearly present even in those who have never been ill. (c) These are maps of cortical thickness with measures of inattention, with cool and warm colors representing significant inverse and positive correlations, respectively. Inverse correlations are present along the entire extent of the lateral surface of the right hemisphere and the medial wall of the left, as well as the posterior portion of the lateral surface of the left hemisphere. In those regions, greater degrees of inattention accompany progressively thinner cortices. This same pattern of correlation was detected in the high-risk group alone, the low-risk group alone, and in both 
groups combined, indicating that this relationship of cortical thinning and its regional pattern is likely to exist in the general population regardless of the degree of familial risk present in any person. $(d)$ These are maps of cortical thickness with measures of performance on tasks on the Wechsler Memory Scales that require processing and recall of visual memory for social stimuli (mostly cartoon-type drawings that require recall of the identity of faces and social scenes). Cool and warm colors represent significant inverse and positive correlations, respectively. Poorer memory performance (lower scores) accompanies thinner cortices (i.e., correlations are positive) along the lateral surface of the right hemisphere and the medial wall of the right, in both the high- and low-risk groups separately and in both groups combined, indicating that this pattern of correlation obtains regardless of the degree of familial risk for MDD. The same spatial pattern of correlation as the pattern for our MDD endophenotype indicates that the cortical thinning in the high-risk group accounts for their poor visual recall for social stimuli. SG, subgenual cortex; OF, orbitofrontal cortex; PC, posterior cingulate. 

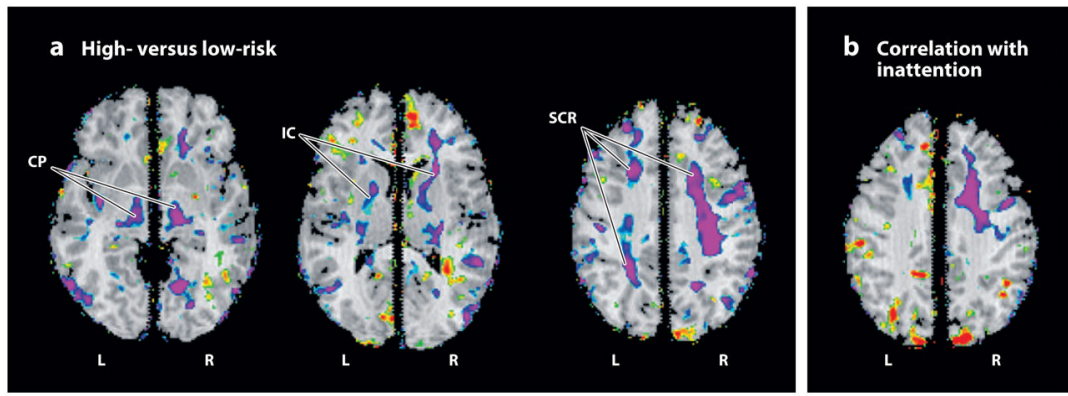

Figure 2.

Maps of local white matter volume. The statistical significance (probability values) of analyses for measures of local volumes of brain tissue, covarying for age and sex, are colorcoded at each point within brain parenchyma. (a) Volumes are compared across high- and low-risk groups. Significantly reduced volumes (purple) are present in the high-risk group, primarily in bilateral dorsal frontal cortices, including the superior corona radiata (SCR) and internal capsule (IC), and in the cerebral peduncles (CP). (b) This is a map of the correlations of local volumes with measures of inattention. Regions of significant inverse correlation (purple) indicate that more inattention accompanies the reduced local volumes present in the high-risk group, primarily within the dorsal portion of the frontal white matter in the right cerebral hemisphere. L, left; R, right. 


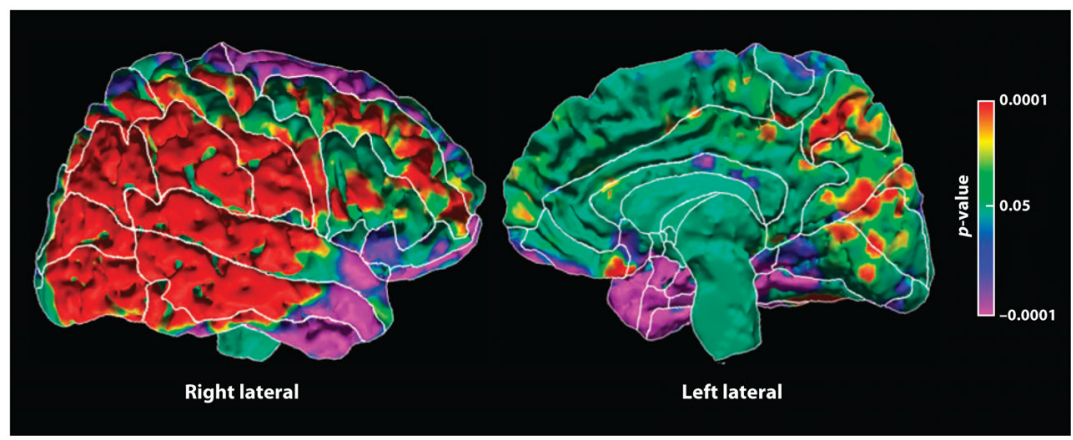

Figure 3.

Correlation of average local white matter volumes with cortical thickness. At each point on the surface of the brain, the average value of the local white matter volumes in the region of frontal and parietal hypoplasia for each person was correlated with the thickness of the cortical mantle at that point. Highly significant positive correlations were found in the posterior aspect of the right hemisphere, in the region of cortical thinning in our major depressive disorder (MDD) endophenotype. These correlations were found in both the highand low-risk groups, indicating that they are independent of risk status. The correlations suggest that these regions of frontal white matter and posterior lateral cortex of the right hemisphere constitute a neural circuit. Reduced volumes in both the cortical and white matter portions of that circuit in our MDD endophenotype suggest that this biomarker affects an entire neural circuit or set of circuits within the brain. 

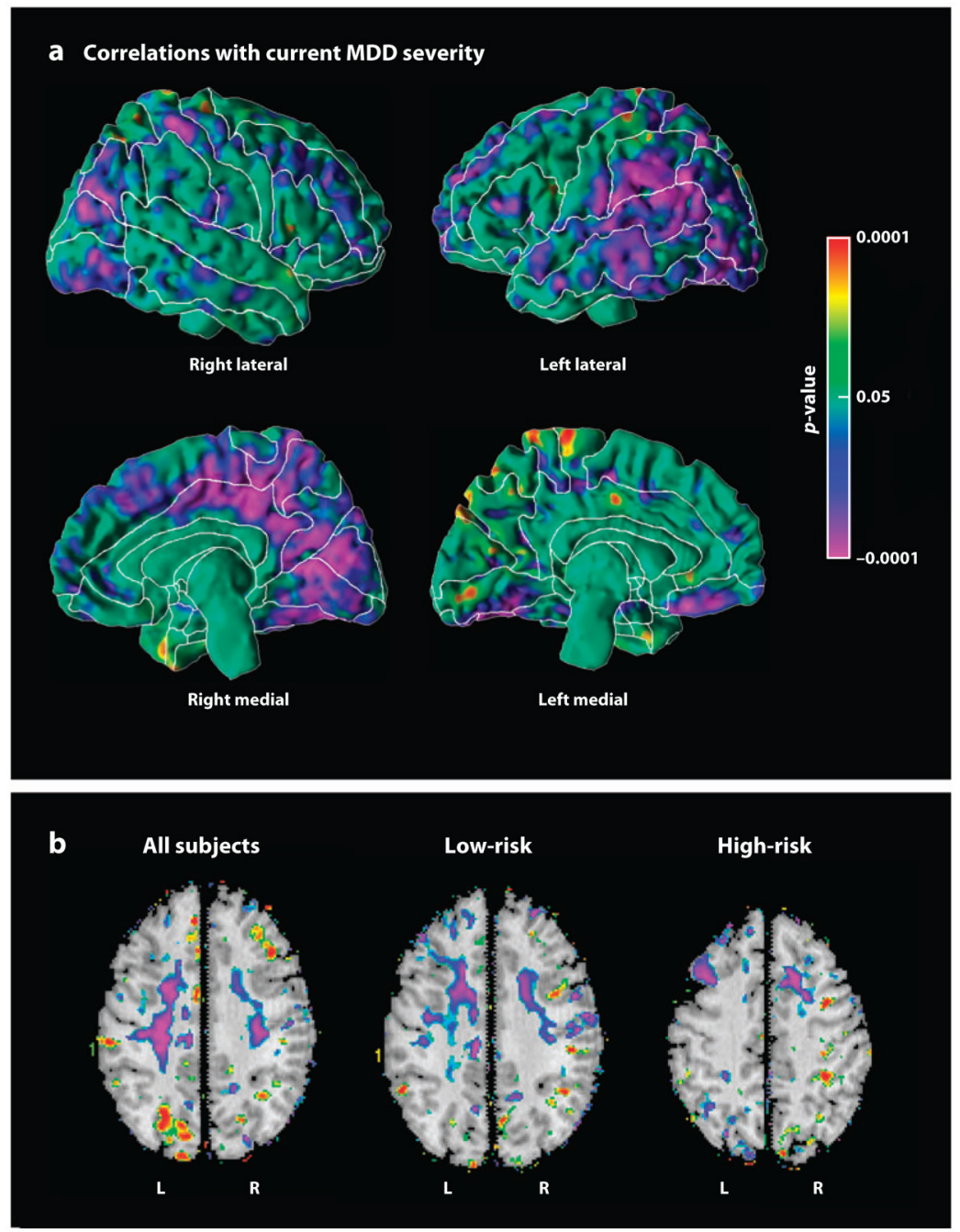

Figure 4.

Correlations with symptom severity at the time of scan. These maps show the correlations of cortical thickness or local volumes with the summed severity of anxiety and depressive symptoms at the time of MRI scan. (a) Cortical thickness correlates inversely (purple) with symptom severity, indicating that more severe symptoms of major depressive disorder (MDD) accompany thinner cortices, primarily in the posterior aspect of the lateral surface of the left hemisphere and the medial wall of the right hemisphere. Similar findings were obtained in each risk group separately (not shown). (b) Local volumes correlate inversely (purple) with symptom severity, primarily within white matter of the dorsal frontal cortices of both hemispheres. 